\title{
Changes in Octaethyleneglycol Mono N-Dodecyl Ether Micelles in Aqueous 1,4-Dioxane Solutions
}

\author{
Hideki Aizawa* \\ Faculty of Pharmaceutical Sciences, Setsunan University, 573-0101,Osaka, Japan
}

\begin{abstract}
The structures of micelles of the surfactant Octaethyleneglycol mono n-dodecyl ether $\left(\mathrm{C}_{12} \mathrm{E}_{8}, \mathrm{BL}-8 \mathrm{SY}\right)$ in $0-80 \%$ aqueous 1,4-dioxane solutions ( $\mathrm{pH} 7.2$, ionic strength $2.44 \mathrm{mM}$ ) were investigated by small-angle X-ray scattering. $\mathrm{C}_{12} \mathrm{E}_{8}$ forms the core-shell discus micelle in $0-80 \%$ aqueous 1,4-dioxane solutions. Changing properties of aqueous 1,4-dioxane solution did not affect the outside-shapes of $\mathrm{C}_{12} \mathrm{E}_{8}$ micelles; however, it might be resulted in changing the structures of $\mathrm{C}_{12} \mathrm{E}_{8}$ micelles from normal core-shell discus micelles to reverse core-shell discus micelles in 1,4-dioxane concentrations between 40 and $60 \%$.
\end{abstract}

Keywords: Micelle, Small-angle X-ray scattering, Micellar structure investigations, Least-squares fit calculation, Ternary system, core-shell discus micelle.

\section{INTRODUCTION}

In aqueous solution, surfactants form micelles with a hydrophobic core and a hydrophilic shell because surfactants are amphipathic, that is, they contain both a hydrophobic group and a hydrophilic group. The properties of surfactant micelles in aqueous solution have been investigated extensively, but there has been little research on the behavior of micelles in aqueous solutions of water-soluble polar organic solvents such as ethylene glycol, glycerol, formamide, and hydrazine [1-5]. In those solvents, mixtures of ethylene glycol and water and those of glycerol and water have been mainly studied. However, there has been extremely little research on clarifying how surfactant molecules formed micelles, and how changing the solvent from $100 \%$ water to a mixture of water and each polar solvent affected the shape and size of micelles.

1,4-dioxane is another polar solvent. The reason why we especially have an interest in 1,4-dioxane as a research target is that aqueous solutions of 1,4-dioxane are normally used as the reference solutions for gauging the apparent water activity and micropolarity of aqueous environments in surfactant self-assembly systems by means of the fluorescent probe method [6].

In recent work, we studied changes in the structure of micelles of the surfactant polysorbate 80 (Tween 80) in 0 $50 \%$ aqueous polar 1,4-dioxane solutions $(\mathrm{pH} \mathrm{7.2,} \mathrm{ionic}$ strength $2.44 \mathrm{mM}$ ) by means of small-angle X-ray scattering (SAXS). Polysorbate 80 contains hydrophilic groups (4 polyoxyethylene groups and tetrahydrofuran group) and hydrophobic group (polyethylene group). We found the 2 phenomena that polysorbate 80 micelles form core-shell cylinder micelles; they change from core-shell cylinder

*Address correspondence to this author at the Faculty of Pharmaceutical Sciences, Setsunan University, 45-1 Nagaotouge-machi Hirakata-City, Osaka, Japan, 573-0101; Tel/Fax: +81 (72) 866-3151;

E-mail: aizawa@pharm.setsunan.ac.jp micelles to core-shell discus micelles between concentrations of 20 and 30\% 1,4-dioxane, and from core-shell discus micelles to core-shell elliptic discus micelles between concentrations of 40 and 50\% 1,4-dioxane [7]. In addition, formation from molecules of polysorbate 80 to core-shell cylinder micelles describes the following description, and it illustrated in Fig 3 of Aizawa [7]. The molecules of polysorbate 80 adopted crown-like shapes. When two molecules of them connected each other, polyethylene group (hydrophobic group) of one molecule went into the space among 4 polyoxyethylene groups (hydrophilic groups) of another molecule one another; The molecules aggregated into a 'cylindrical' layer of four long chains entangled with one another through intra- and intermolecular interactions. It is natural that the division between hydrophobicity and hydrophilicity exists in the inner micelle. The outer core-shell cylinder is covered with hydrophilic groups. Therefore, the core-shell cylinder micelles are stable in aqueous solution.

Octaethyleneglycol mono n-dodecyl ether $\left[\mathrm{C}_{12} \mathrm{H}_{25}\right.$ $\left.\left(\mathrm{OC}_{2} \mathrm{H}_{4}\right)_{8} \mathrm{OH}, \mathrm{C}_{12} \mathrm{E}_{8}, \mathrm{BL}-8 \mathrm{SY}\right]$ is another type of surfactant, simple structure of a linear chain that contains two moieties of hydrophobic polyethylene and hydrophilic polyoxyethylene (See Fig. 1) [8], and thus it is easy to clarify how molecules of $\mathrm{C}_{12} \mathrm{E}_{8}$ form micelles, and how changing the solvent from $100 \%$ water to a mixture of water and 1,4dioxane affect the shape and size of $\mathrm{C}_{12} \mathrm{E}_{8}$ micelles. Such information can further our understanding of the underlying basic principles of micelle formation and morphology.

In this study, we investigated the structure of $\mathrm{C}_{12} \mathrm{E}_{8}$ micelles in aqueous 1,4-dioxane ( $\mathrm{pH} 7.2$, ionic strength 2.44 $\mathrm{mM}$ ) by means of SAXS.

\section{MATERIALS AND METHODOLOGY}

\section{Chemicals and Sample Preparation}

Octaethyleneglycol mono n-dodecyl ether $\left(\mathrm{C}_{12} \mathrm{E}_{8}\right.$, commercial name BL-8SY) was obtained from Nikko Chemicals 


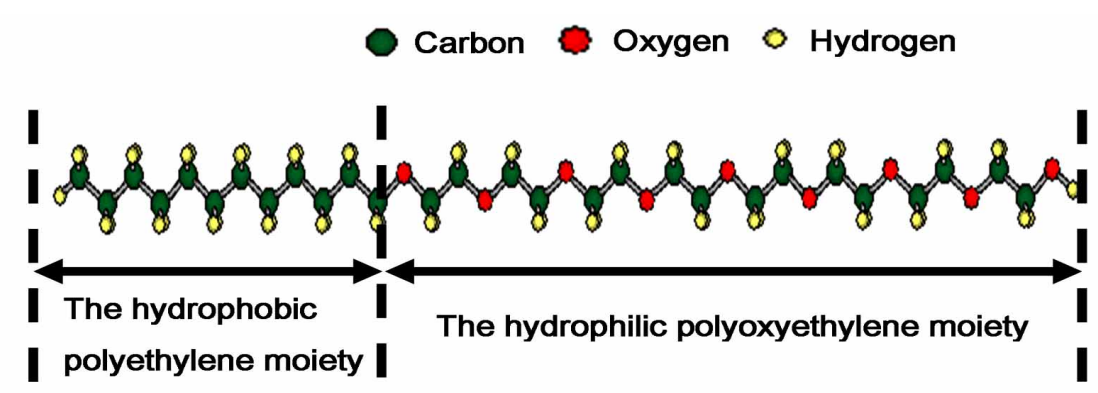

Ball-and-stick model of the $\mathrm{C}_{12} \mathrm{E}_{8}$ molecule

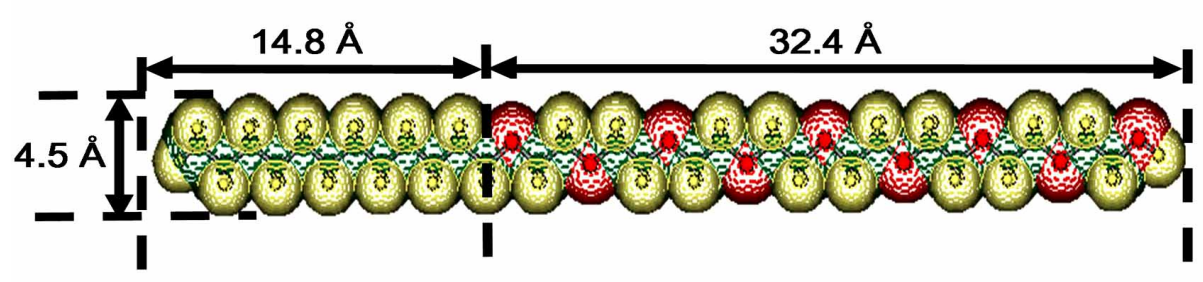

Space-filling model of the $C_{12} E_{8}$ molecule

Fig. (1). Typical $\mathrm{C}_{12} \mathrm{E}_{8}$ molecule molecular structure.

Co. (Tokyo, Japan). 1,4-Dioxane was obtained from Nacalai Tesque (Kyoto, Japan), and disodium hydrogen phosphate $\left(\mathrm{Na}_{2} \mathrm{HPO}_{4} \cdot 12 \mathrm{H}_{2} \mathrm{O}\right)$ and sodium dihydrogen phosphate $\left(\mathrm{NaH}_{2} \mathrm{PO}_{4} \cdot 2 \mathrm{H}_{2} \mathrm{O}\right)$ were obtained from Wako Pure Chemical Industries (Osaka, Japan).

A $10 \mathrm{mM}$ stock solution of sodium phosphate buffer ( $\mathrm{pH}$ 7.2 , ionic strength $24.4 \mathrm{mM}$ ) was prepared by dissolving a mixture of $7.2 \mathrm{mmol} \mathrm{Na}_{2} \mathrm{HPO}_{4} \cdot 12 \mathrm{H}_{2} \mathrm{O}$ and $2.8 \mathrm{mmol}$ $\mathrm{NaH}_{2} \mathrm{PO}_{4} \cdot 2 \mathrm{H}_{2} \mathrm{O}$ in $1 \mathrm{~L}$ of water. A $500 \mathrm{~g} \cdot \mathrm{L}^{-1}$ stock solution of $\mathrm{C}_{12} \mathrm{E}_{8}$ was prepared by dissolving $\mathrm{C}_{12} \mathrm{E}_{8}$ in the stock sodium phosphate buffer. Sample solutions containing 0-80\% 1,4-dioxane, $50 \mathrm{~g} \cdot \mathrm{L}^{-1} \mathrm{C}_{12} \mathrm{E}_{8}$, and $1 \mathrm{mM}$ sodium phosphate buffer ( $\mathrm{pH} \mathrm{7.2,} \mathrm{ionic} \mathrm{strength} 2.44 \mathrm{mM}$ ) were prepared by mixing 1,4-dioxane, the stock sodium phosphate buffer, the stock $\mathrm{C}_{12} \mathrm{E}_{8}$ solution, and water. Reference solutions containing $0-80 \%$ 1,4-dioxane and $1 \mathrm{mM}$ sodium phosphate buffer were prepared by mixing 1,4-dioxane, the stock sodium phosphate buffer, and water.

\section{SAXS Measurements and Analysis of SAXS Data}

Small-angle X-ray scattering (SAXS) equipment for solution analysis, including optics and a detector system, were used for this study. This equipment is installed at the Energy Accelerator Research Organization in Tsukuba, Japan, and the specifications of this equipment are detailed in Kajiwara $\&$ Hiragi and Ueki $[9,10]$. SAXS intensities were measured for $900 \mathrm{~s}$ for each surfactant solution and for a reference solution, and intensities were calibrated and transformed into scattering cross-sections $\{\operatorname{SCS}(q)$, where $q$ is the scattering vector $[q=(4 \pi / \lambda) \sin \theta$ (where $\lambda$ is the wavelength of the $\mathrm{X}$ rays and $2 \theta$ is the scattering angle)]\} based on the same method presented by Aizawa [7].

We attempted to fit the SAXS data to each of the following alternative models: the monodisperse core-shell sphere, the polydisperse sphere, the core-shell tri-axial ellipsoid (Structure factor of each model is a hard-sphere potential energy model), the core-shell cylinder model for dilute particle solutions, the core-shell cylinder for concentrated particle solutions by means of each of random phase approximation (RPA) and polymer reference interaction site model (PRISM) [11-13]. Least-squares fit calculations showed that the core-shell cylinder model of dilute particle solutions provided the best fit for the SAXS data at 1,4-dioxane concentrations of $0-80 \%$.

The mathematical description of the core-shell cylinder model for dilute particle solutions is given below [7].

$\operatorname{SCS}(q)$, which does not take into account the structure factor (in dilute particle solutions), is given by

$\operatorname{SCS}(q)=n V^{2} \Delta \beta^{2} F(q)^{2}$

where $n, \Delta \beta$, and $V$ are the number density of particles, the difference in scattering length density between the particles and the solvent or matrix, and the volume of particles, respectively; and $F(q)$ is the form factor.

For the core-shell cylinder, $V$ and $\Delta \beta^{2} F(q)^{2}$ are given by

$V=\pi(R+d)^{2} H$

and

$\Delta \beta^{2} F(q)^{2}=\int_{0}^{1}\left\{\left[C F_{1}(q)+C F_{2}(q)\right] / C F_{3}(q)\right\}^{2} \mathrm{~d} x$

where

$C F_{I}(q)=\left\{4 R\left(\beta_{\text {core }}-\beta_{\text {shell }}\right) J_{1}\left[q R\left(1-x^{2}\right)^{1 / 2}\right]\right.$

$\sin (q H x / 2)\} /\left[q^{2} x\left(1-x^{2}\right)^{1 / 2}\right]$

and

$C F_{2}(q)=\left\{4(R+d)\left(\beta_{\text {shell }}-\beta_{\text {solvent }}\right) J_{1}[q(R+d)\right.$

$\left.\left.\left(1-x^{2}\right)^{1 / 2}\right] \sin (q H x / 2)\right\} /\left[q^{2} x\left(1-x^{2}\right)^{1 / 2}\right]$

and

$C F_{3}(q)=R^{2} H\left(\beta_{\text {core }}-\beta_{\text {shell }}\right)+(R+d)^{2} H\left(\beta_{\text {shell }}-\beta_{\text {solvent }}\right)$ 
where $R$ and $\beta_{\text {core }}$ are the radius of the circular base of the core cylinder and the scattering length density of the core, respectively; $d$ and $\beta_{\text {shell }}$ are the length and the scattering length density of the shell, respectively; $H$ is the height of the core-shell cylinder; $\beta_{\text {solvent }}$ is the scattering length density of the solvent; $J_{1}(\mathrm{x})$ is first order Bessel function and $x$ is distance on the $x$ axis.

Six parameters $\left(n, R, d, H, \beta_{\text {core }}\right.$, and $\left.\beta_{\text {shell }}\right)$ for equations (1) to (6) were determined by least-squares fit of the SAXS data to the model. Rough values of $\beta_{\text {core }}$ and $\beta_{\text {shell }}$ were estimated and the exact value of $\beta_{\text {solvent }}$ was determined from the density and the number of electrons of $\mathrm{C}_{12} \mathrm{E}_{8}$ and the mixed solvents [12]. $\beta_{\text {core }}=\left(\rho_{\text {core }} \mathrm{Ne}_{\text {core }} \mathrm{Th} \mathrm{N}_{\mathrm{A}}\right) / \mathrm{M}_{\text {core }}$, where $\rho_{\text {core }}$ :density of the core, $\mathrm{Ne}_{\text {core }}$ eelectron number of molecule of core, $\mathrm{N}_{\mathrm{A}}$ : Adgadro Number, Th:Thomson radius, $\mathrm{M}_{\text {core }}$ : molecular weight of core. $\beta_{\text {shell }}=\left(\rho_{\text {shell }} \mathrm{Ne}_{\text {shell }} \mathrm{Th} \mathrm{N}_{\mathrm{A}}\right) / \mathrm{M}_{\text {shell }}$, where $\rho_{\text {shell }}$ :density of the shell, $\mathrm{Ne}_{\text {shell }}$ :electron number of molecule of shell, $\mathrm{N}_{\mathrm{A}}$ : Adgadro Number, Th:Thomson radius, $M_{\text {shell }}:$ molecular weight of shell. $\beta_{\text {solvent }}=\left(\rho_{\text {solvent }} \mathrm{Ne}_{\text {sol- }}\right.$ vent $\mathrm{Th}_{\mathrm{A}}$ ) / $\mathrm{M}_{\text {solvent }}$, where $\rho_{\text {solvent }}$ :density of the solvent, $\mathrm{Ne}$ shell:electron number of molecule of solvent, $\mathrm{N}_{\mathrm{A}}$ : Adgadro Number, Th:Thomson radius, $\mathrm{M}_{\text {solvent }}$ : molecular weight of solvent. The density $\left(0.974 \mathrm{~g} \cdot \mathrm{cm}^{-3}\right)$ of $\mathrm{C}_{12} \mathrm{E}_{8}$ was determined with an electric balance and those of the reference solvents were determined with a pycnometer. The scattering length density $\left(9.084 \mu \AA^{-2}\right)$ of $C_{12} E_{8}$ and those of the reference solvents were calculated from the above equation for $\beta_{\text {solvent }}$. The densities and scattering length densities are listed in Table 1. The least-squares fit calculation methods for the model and the SAXS data are detailed in Aizawa [7].

\section{RESULTS AND DISCUSSION}

\section{Formation of $C_{12} E_{8}$ Micelle}

As the end result of our attempt to fit the abovealternative models to the SAXS data, the core-shell cylinder model of dilute particle solutions provided the best fit for the SAXS data at 0-80\% 1,4-dioxane. Scattering data obtained at 1,4-dioxane concentrations between 0 and $80 \%$ are shown Fig. (2), along with a curve fitted with the core-shell cylinder. The 1,4-dioxane concentrations and shape parameters calculated from the core-shell cylinder in dilute particle solution are listed in Table 2. At 1,4-dioxane concentrations of $0-80 \%$, because $(R+d)>H$ (See the data in Table 2), the $\mathrm{C}_{12} \mathrm{E}_{8}$ micelles were concluded to be core-shell discus micelles rather than core-shell cylindrical micelles.

Practically, normal SAXS users cannot perform the leastsquares fit calculation for the above-alternative models (especially, polydisperse sphere, core-shell tri-axial ellipsoid and the core-shell cylinder) to the SAXS data because it is extremely difficult for them to make the programs for the least-squares fit calculation. The author overcame the difficulties and obtained the most recent discoveries of the for- mation of $\mathrm{C}_{12} \mathrm{E}_{8}$ micelle, and then considered that it is significant to announce the results to public.

Space-filling model of the $\mathrm{C}_{12} \mathrm{E}_{8}$ molecule (See Fig. 1) shows that the height of $\mathrm{C}_{12} \mathrm{E}_{8}$ molecule is $4.5 \AA$, and the length of moiety of the hydrophobic polyethylene is $14.8 \AA$, and the length of moiety of the hydrophilic polyoxyethylene is $32.4 \AA$, and the total length of the $\mathrm{C}_{12} \mathrm{E}_{8}$ molecule is 47.2 $\AA$. At $0 \%$ 1,4-dioxane concentration, the hydrophobic core in core-shell discus micelle $(R=15.7 \AA)$ was longer than the length of moiety of the hydrophobic polyethylene, and the hydrophilic shell in core-shell discus micelle $(d=18.8 \AA)$ was shorter than the length of moiety of the hydrophilic polyoxyethylene. The $\mathrm{C}_{12} \mathrm{E}_{8}$ molecules (See Fig. 3) aggregated like a discus and they curled up owing to the combined effect of the properties of the mixed solvent and intermolecular interactions between $\mathrm{C}_{12} \mathrm{E}_{8}$ molecules. These processes resulted in the formation of the core-shell discus micelle.

The hydrophobic interface (moiety of polyethylene) exist central area of interface on top and base of the core-shell discus micelle and they are bare to water. The only problem is that the hydrophobic interface are high-thermodynamically energetic unfavorable. However, it is no problem because the interface of both hydrophobicity (moiety of polyethylene) and hydrophilicity (moiety of polyoxyethylene) on the outer core-shell discus micelle are covered with hydrogen atoms, which can connect with oxygen of water molecules by hydrogen bond. Thus, the core-shell discus micelles of $\mathrm{C}_{12} \mathrm{E}_{8}$ are thermodynamically energetic favorable.

\section{Change in Formation of $\mathrm{C}_{12} \mathrm{E}_{8}$ Micelle as 1,4-Dioxane Concentration Increased}

The shapes of $\mathrm{C}_{12} \mathrm{E}_{8}$ micelles were the same at $0-80 \%$ 1,4-dioxane concentrations; that is, the micelle shape did not change as 1,4-dioxane concentration increased. Changing properties of 1,4-dioxane mixture did not affect the outsideshape of $\mathrm{C}_{12} \mathrm{E}_{8}$ micelles. In contrast, the radii of the circular base of the core discus $(R)$ (See the data in Table 2 ) were 15 $\AA$ or thereabouts at $0-40 \%$ 1,4-dioxane concentrations, however, the radius of the circular base of the core discus $(R)$ rapidly decreased at $60 \%$ 1,4-dioxane concentration. The lengths of the shell of the circular base of the core-shell discus $(d)$ decreased at 0-40\% 1,4-dioxane concentrations, however, the length of the shell of the circular base of the core-shell discus $(d)$ rapidly increased at $60 \%$ 1,4-dioxane concentration. The heights of the core-shell discus $(H)$ were $13 \AA$ or thereabouts at 0-80\% 1,4-dioxane concentrations. Thus, their changes indicate the inner structure of $\mathrm{C}_{12} \mathrm{E}_{8}$ micelles changed in 1,4-dioxane concentrations between 40 and $60 \%$.

The $\mathrm{C}_{12} \mathrm{E}_{8}$ molecule contains two moieties of hydrophobic polyethylene and hydrophilic polyoxyethylene. If $\mathrm{C}_{12} \mathrm{E}_{8}$ molecules dissolve in aqueous solutions, they form normal micelles (hydrophobic-core/hydrophilic-shell micelles). In contrast, if they dissolve in organic solvents,

Table 1. Densities and Scattering Length densities of the Reference Solvent

\begin{tabular}{|l|l|l|l|l|l|}
\hline $1,4-$ Dioxane concentration $(\%)$ & 0 & 20 & 40 & 60 & 80 \\
\hline Density $\left(\mathrm{g} \cdot \mathrm{cm}^{-3}\right)$ & 0.996 & 1.014 & 1.028 & 1.036 & 1.035 \\
\hline Scattering length density $\left(\mu \AA^{-2}\right)$ & 9.216 & 9.826 & 10.44 & 10.96 & 11.39 \\
\hline
\end{tabular}




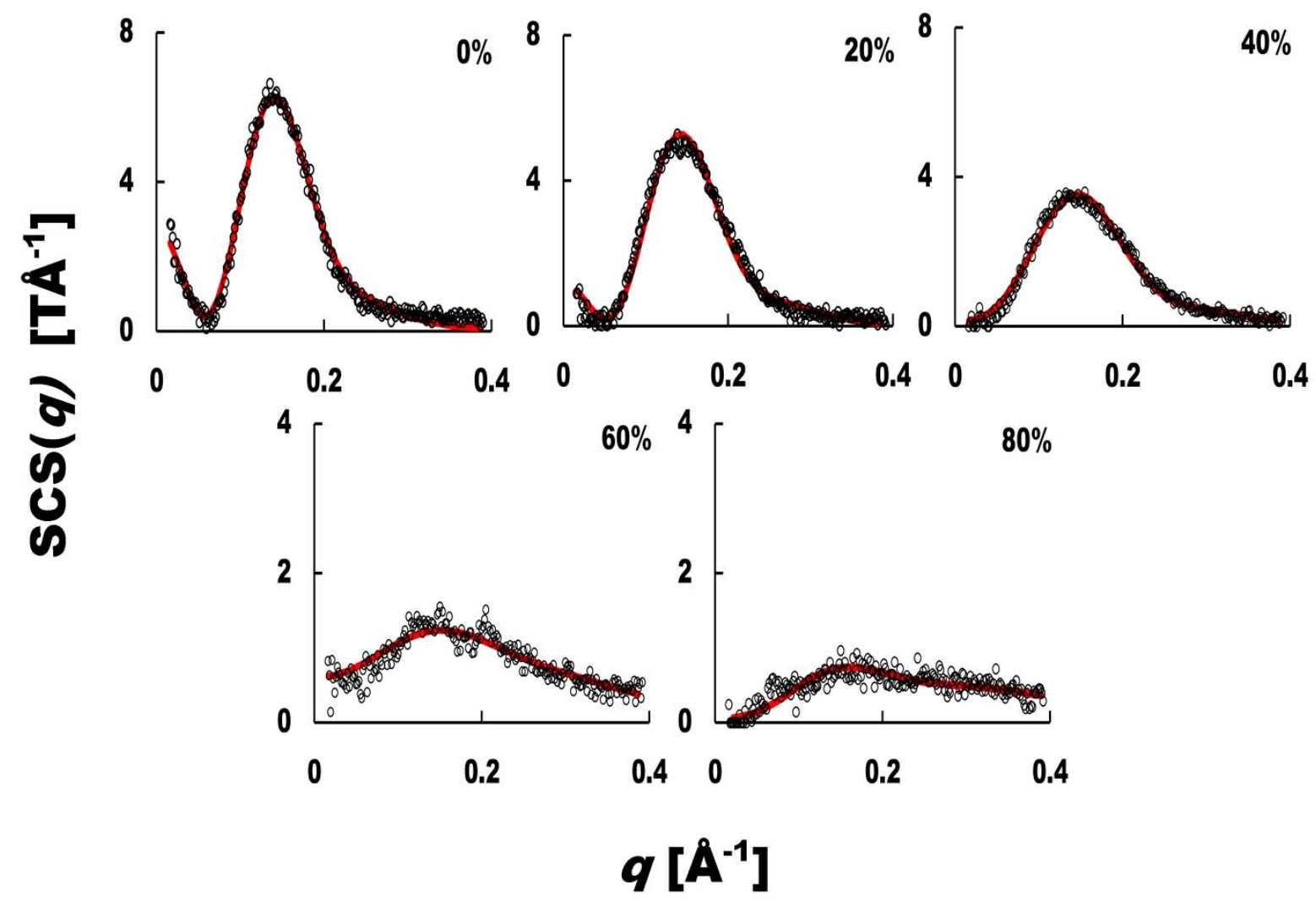

Fig (2). Scattering data (circles) obtained at 1,4-dioxane concentrations between 0 and $80 \%$, along with curves fitted with core-shell cylinder model: $\operatorname{SCS}(q)=1$ (red lines).

Table 2. Shape Parameters for the Core-Shell Cylinder Model for Dilute Particle Solutions at Various 1,4-Dioxane Concentrations

\begin{tabular}{|l|l|l|l|l|l|l|}
\hline $\begin{array}{l}\text { Conc. } \\
(\%)\end{array}$ & $\begin{array}{l}n \\
\left(\mathbf{a}^{-3}\right)\end{array}$ & $\begin{array}{l}\boldsymbol{R} \\
(\mathbf{\AA})\end{array}$ & $\begin{array}{l}\boldsymbol{d} \\
(\mathbf{\AA})\end{array}$ & $\begin{array}{l}\boldsymbol{H} \\
(\mathbf{\AA})\end{array}$ & $\begin{array}{l}\boldsymbol{\beta}_{\text {core }} \\
\left(\boldsymbol{\mu} \AA^{-2}\right)\end{array}$ & $\begin{array}{l}\boldsymbol{\beta}_{\text {shell }} \\
\left(\boldsymbol{\mu} \AA^{-2}\right)\end{array}$ \\
\hline \hline 0 & 52 & 15.7 & 18.8 & 14.2 & 8.026 & 9.697 \\
\hline 20 & 24 & 15.9 & 17.3 & 14.4 & 8.636 & 10.31 \\
\hline 40 & 3 & 14.9 & 15.0 & 12.0 & 6.208 & 11.67 \\
\hline 60 & 15 & 5.4 & 27.5 & 11.8 & 6.095 & 11.01 \\
\hline 80 & 1 & 3.0 & 32.2 & 12.3 & 5.982 & 11.42 \\
\hline
\end{tabular}

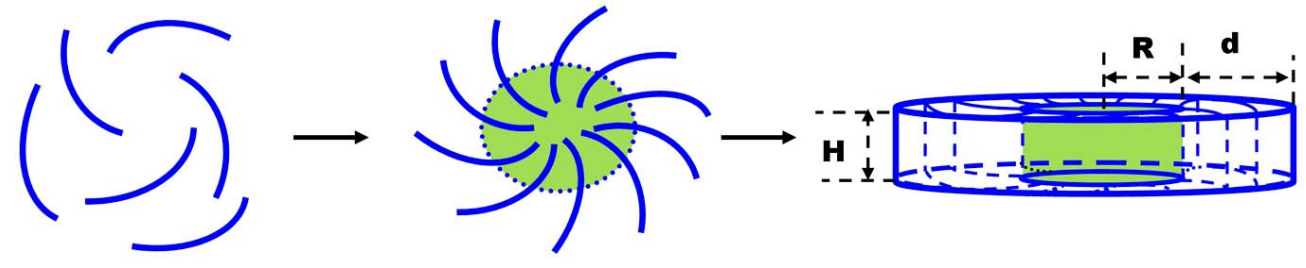

Monomers

Discus-like aggregation

Core shell discus micelle

Fig (3). Formation from $\mathrm{C}_{12} \mathrm{E}_{8}$ molecules to $\mathrm{C}_{12} \mathrm{E}_{8}$ micelles.

they form reverse micelles (hydrophilic-core/hydrophobicshell micelles). Thus, the changes in the structures of $\mathrm{C}_{12} \mathrm{E}_{8}$ micelles in 1,4-dioxane concentrations between 40 and $60 \%$ might be transition from normal core-shell discus micelles to reverse core-shell discus micelles. If reverse core-shell discus micelles formed in aqueous solutions of high 1,4dioxane concentrations, it is also no problem about the mi- celle structure because the interface of both hydrophobicity (moiety of polyethylene) and hydrophilicity (moiety of polyoxyethylene) on the outer core-shell discus micelle are covered with hydrogen atoms, which can connect with oxygen of water molecules by hydrogen bond. Thus, the core-shell discus micelles of $\mathrm{C}_{12} \mathrm{E}_{8}$ are thermodynamically energetic favorable. 
Although " $\mathrm{C}_{12} \mathrm{E}_{8}$ micelles in aqueous 1,4-dioxane solution are the core-shell discus micelles" is presented as a new discovery, normal colloidal scientists believe that "Hydrodynamic stable shape in aqueous solutions is sphere or ellipsoid like bubbles, and thus normal shape of the micelles is also sphere or ellipsoid." as a common sense. The only problem is that which shape of the micelles is thermodynamically energetic favorable in aqueous 1,4-dioxane solutions - sphere, ellipsoid, or core-shell discus. Molecular dynamic simulation would be best solution to the problem and provide corroboration for the above results. However, the author does not have the knowledge about how to perform it and could not find it in many literatures as long as we checked. We are seeking co-researchers who have the skill in molecular dynamic simulation.

\section{CONCLUSIONS}

The structures of micelles of the surfactant Octaethyleneglycol mono n-dodecyl ether $\left(\mathrm{C}_{12} \mathrm{E}_{8}, \mathrm{BL}-8 \mathrm{SY}\right)$ in $0-80 \%$

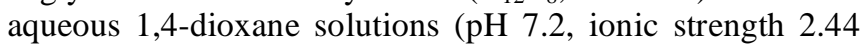
$\mathrm{mM}$ ) were investigated by small-angle X-ray scattering. They might be changed from normal core-shell discus micelles (hydrophobic-core and hydrophilic-shell micelles) to reverse core-shell discus micelles (hydrophilic-core and hydrophobic-shell micelles) in 1,4-dioxane concentrations between 40 and $60 \%$.

\section{REFERENCES}

[1] Ramadan, M.S.; Evans, D.F.; Lumry, R.; Philson, S. Micelle formation in hydrazine-water mixtures. J. Phys. Chem., 1985, 89(15), 3405-3408.
[2] Lattes, A.; Rico, I. Aggregation in formamide solution: Reactivity and structure of non-aqueous microemulsions. Colloids Surf., 1989, 35(2), 221-235.

[3] Rodriguez, A.; Graciani, M.M.; Muñoz, M.M.; Moyá, M. L. Water-ethylene glycol alkyltrimethylammonium bromide micellar solutions as reaction media: study of spontaneous hydrolysis of phenyl chloroformate. Langmuir., 2003, 19(18), 7206-7213.

[4] D'Errico, G.; Ciccarelli, D.; Ortona, O. Effect of glycerol on micelle formation by ionic and nonionic surfactants at $25{ }^{\circ} \mathrm{C} . J$. Colloid Interface Sci., 2005, 286(2), 747-754.

[5] Fanun, M.; Microemulsions with Nonionic Surfactants and Mint Oil. Open Colloid Sci. J., 2010, 3, 9-14.

[6] Chakrabarty, A.; Das, P.; Mallick, A.; Chattopadhyay, N. Effect of surfactant chain length on the binding interaction of a biological photosensitizer with cationic micelles. J. Phys. Chem. B., 2008, 112(12), 3684-3692.

[7] Aizawa, H. Morphology of polysorbate 80 (Tween 80) micelles in aqueous 1,4-dioxane solutions. J. Appl. Cryst., 2009, 42(4), 592-596.

[8] Senda, N. Winmostar, 3D-Graphics program for Molecular Modeling and Visualization of Quantum Chemical Calculations; Tencube Labs.:Chiba, Japan, 2002.

[9] Kajiwara, K.; Hiragi, Y. Structure Analysis by Small-Angle X-Ray Scattering. In Saisho, H.; Gohshi, Y., Eds.; Applications of Synchrotron Radiation to Materials Analysis; Elsevier Science B.V.: Amsterdam, 1996, pp. 353-404.

[10] Ueki, T. Biopolymers and solution X-ray scattering Is it useful in structural research in biolory? Nucl. Instrum. Methods. Phys. Res., Sect. A, 1991, A303, 464-475.

[11] Pedersen, J.S. Analysis of small-angle scattering data from colloids and polymer solutions: modeling and least-squares fitting. Adv. Colloid Interface Sci., 1997, 70, 171-210.

[12] Lindner, P.; Zemb, T, Eds. Neutron, X-rays and Light Scattering Methods Applied to Soft Condensed Matter; Elsevier Science: Amsterdam, 2002.

[13] Aizawa, H. Method for estimating particle shape in liquid. JP Patent 4598899, 2010 or PCT Pat. Appl., PCT/JP2010/050187, 2010. 